\title{
Linking pangenomes and metagenomes: the prochlorococcus metapangenome
}

\author{
Tom 0 Delmont ${ }^{\text {Corresp., } 1}$, A. Murat Eren ${ }^{\text {Corresp. } 1,2}$ \\ 1 Department of Medicine, University of Chicago, Chicago, Illinois, United States \\ 2 Josephine Bay Paul Center, Marine Biological Laboratory, Woods Hole, MA, United States \\ Corresponding Authors: Tom O Delmont, A. Murat Eren \\ Email address: tomodelmont@gmail.com, a.murat.eren@gmail.com
}

Pangenomes offer detailed characterizations of core and accessory genes found in a set of closely related microbial genomes, generally by clustering genes based on sequence homology. In comparison, metagenomes facilitate highly resolved investigations of the relative distribution of microbial genomes and individual genes across environments through read recruitment analyses. Combining these complementary approaches can yield unique insights into the functional basis of microbial niche partitioning and fitness, however, advanced software solutions are lacking. Here we present an integrated analysis and visualization strategy that provides an interactive and reproducible framework to generate pangenomes and to study them in conjunction with metagenomes. To investigate its utility, we applied this strategy to a Prochlorococcus pangenome in the context of a large-scale marine metagenomic survey. The resulting Prochlorococcus metapangenome revealed remarkable differential abundance patterns between very closely related isolates that belonged to the same phylogenetic cluster and that differed by only a small number of gene clusters in the pangenome. While the relationships between these genomes based on gene clusters correlated with their environmental distribution patterns, phylogenetic analyses using marker genes or concatenated single-copy core genes did not recapitulate these patterns. The metapangenome also revealed a small set of core genes that mostly occurred in hypervariable genomic islands of the Prochlorococcus populations, which systematically lacked read recruitment from surface ocean metagenomes. Notably, these core gene clusters were all linked to sugar metabolism, suggesting potential benefits to Prochlorococcus from a high sequence diversity of sugar metabolism genes. The rapidly growing number of microbial genomes and increasing availability of environmental metagenomes provide new opportunities to investigate the functioning and the ecology of microbial populations, and metapangenomes can provide unique insights for any taxon and biome for which genomic and sufficiently deep metagenomic data are available. 
3 Tom O. Delmont ${ }^{1, *}$ and A. Murat Eren ${ }^{1,2, *}$

$4 \quad{ }^{1}$ Department of Medicine, The University of Chicago, Chicago, IL 60637, United States

$5{ }^{2}$ Josephine Bay Paul Center, Marine Biological Laboratory, Woods Hole, MA 02543, United States

$6 \quad{ }^{*}$ Corresponding authors

7 Tom O. Delmont

$8 \quad$ E-mail address: tomodelmont@gmail.com

11 A. Murat Eren

15 Running Title

16 The Metapangenome of Prochlorococcus

\section{Abstract}

18 Pangenomes offer detailed characterizations of core and accessory genes found in a set

19 of closely related microbial genomes, generally by clustering genes based on sequence

20 homology. In comparison, metagenomes facilitate highly resolved investigations of the

21 relative distribution of microbial genomes and individual genes across environments

22 through read recruitment analyses. Combining these complementary approaches can

23 yield unique insights into the functional basis of microbial niche partitioning and fitness,

24 however, advanced software solutions are lacking. Here we present an integrated

25 analysis and visualization strategy that provides an interactive and reproducible

26 framework to generate pangenomes and to study them in conjunction with

27 metagenomes. To investigate its utility, we applied this strategy to a Prochlorococcus

28 pangenome in the context of a large-scale marine metagenomic survey. The resulting 
29 Prochlorococcus metapangenome revealed remarkable differential abundance patterns

30 between very closely related isolates that belonged to the same phylogenetic cluster and

31 that differed by only a small number of gene clusters in the pangenome. While the

32 relationships between these genomes based on gene clusters correlated with their

33 environmental distribution patterns, phylogenetic analyses using marker genes or

34 concatenated single-copy core genes did not recapitulate these patterns. The

35 metapangenome also revealed a small set of core genes that mostly occurred in

36 hypervariable genomic islands of the Prochlorococcus populations, which systematically

37 lacked read recruitment from surface ocean metagenomes. Notably, these core gene

38 clusters were all linked to sugar metabolism, suggesting potential benefits to

39 Prochlorococcus from a high sequence diversity of sugar metabolism genes. The rapidly

40 growing number of microbial genomes and increasing availability of environmental

41 metagenomes provide new opportunities to investigate the functioning and the ecology

42 of microbial populations, and metapangenomes can provide unique insights for any

43 taxon and biome for which genomic and sufficiently deep metagenomic data are

44 available.

45 Introduction

46 During the last two decades, the genomic content of more than 100,000 microbial

47 isolates has been characterized and used to study the gene pool, adaptation

48 capabilities, and evolution of microorganisms (Smith et al., 1997; Alm et al., 1999;

49 Makarova et al., 2006; Kumar et al., 2011; Fernández-Gómez et al., 2013). Cultivation-

50 based approaches have paved the way for the emergence of powerful strategies to

51 identify core and accessory genes shared between closely related genomes through

52 pangenomics (Read et al., 2003; Tettelin et al., 2005; Zhu et al., 2015). Genomic

53 comparisons of isolates can shed light on the biogeographic partitioning of variable 
54 genes within microbial lineages (Reno et al., 2009; Porter et al., 2016). Yet, de novo

55 investigations of the role of genomic traits in the adaptation of microorganisms to the

56 environment remains difficult to achieve due to our inability to infer distribution patterns

57 of microbial populations based on cultivars.

58 Shotgun metagenomics, the sequencing of DNA directly extracted from the environment

59 (Handelsman et al., 1998), allows the study of microbial communities without the need

60 for cultivation. As of today, metagenomic data originating from a wide range of

61 ecosystems make up a large fraction of the sequences stored in public databases (Qin

62 et al., 2010; Bork et al., 2015). Researchers have used metagenomics to discover new

63 bioactive molecules (Lorenz \& Eck, 2005; Thies et al., 2016), investigate the functional

64 potential of ecosystems (Tringe et al., 2005; Al-Amoudi et al., 2016), and access the

65 genomic context of uncultivated microorganisms (Tyson et al., 2004; Haroon et al., 2016;

66 Delmont et al., 2017). Metagenomic data also provide a means to quantify the

67 abundance and relative distribution of genomes in environmental samples through read

68 recruitment (Tyson et al., 2004; Dutilh et al., 2014; Eren et al., 2015). Although the

69 environmental signal resulting from such analyses provides insights into the ecological

70 niche of individual populations (Sharon et al., 2013; Bendall et al., 2016; Anderson et al.,

71 2017; Quince et al., 2017), this approach alone does not reveal to what extent genes

72 that may be linked to the ecology and fitness of microbes are conserved within a

73 phylogenetic clade.

74 Recently, pangenomic approaches have been used to characterize the gene content of

75 microbial populations in environmental samples through metagenomic read recruitment

76 (Delmont \& Eren, 2016; Scholz et al., 2016; Nayfach et al., 2016). Combining well-

77 established practices from pangenomics (identifying gene clusters and inferring

78 relationships between genomes based on shared genes), with the emerging 
79 opportunities from metagenomics (the ability to track populations precisely across

80 environments through genome-wide read recruitment) could provide a framework to

81 investigate the ecological role of gene clusters that may be linked to the niche

82 partitioning and fitness of microbial populations. To explore the potential of this concept,

83 we developed a novel workflow within an existing open-source software platform (Eren et

84 al., 2015), and characterized the metapangenome of Prochlorococcus isolates and

85 single-cell genomes on a large scale.

86 Prochlorococcus is an extensively studied photosynthetic bacterial taxon abundant in the

87 euphotic zone of low latitude marine systems (Chisholm et al., 1988; Olson et al., 1990;

88 Rusch et al., 2010), which fixes a substantial amount of carbon from the atmosphere

89 (Flombaum et al., 2013). Cultivation efforts targeting Prochlorococcus resulted in the

90 recovery of genomes that represent members from five major phylogenetic clades

91 divided into groups that are adapted to high-light (sub-clades HL-I and HL-II) or low-light

92 (sub-clades LL-I, LL-II, LL-III, and LL-IV) (Biller et al., 2014a). Environmental surveys and

93 culture experiments revealed the ecological niche and temporal dynamics of $\mathrm{HL}$ and $\mathrm{LL}$

94 Prochlorococcus ecotypes in the oceans, as well as correlations between the genomic

95 traits of isolates and their response to environmental variables (West et al., 2001; Rocap

96 et al., 2003; Malmstrom et al., 2010). A previous study by Coleman \& Chisholm

97 (Coleman \& Chisholm, 2010) used a pangenome of 12 Prochlorococcus isolates to

98 discuss the differential occurrence in Prochlorococcus populations between two

99 sampling stations after identifying core versus accessory genes and observing that only

100 a few genes differed significantly in abundance between the sites. In addition, Kent et al.

101 (Kent et al., 2016) showed a strong association between the Prochlorococcus accessory

102 gene functions and the community composition of this lineage on a large scale using

103 metagenomes from the Global Ocean Sampling expedition. Yet, to the best of our

104 knowledge, pangenomes have never been linked to metagenomes at an appropriate 
105 resolution to monitor the distribution of individual gene clusters. Monitoring individual

106 gene clusters is essential to scrutinize their prevalence across multiple microbial

107 genomes, and infer associations regarding their potential role in fitness and niche

108 partitioning of microbial populations to which they belong.

109 Here we investigated the gene clusters we identified in 31 Prochlorococcus isolates in

110 conjunction with their occurrence in the surface of marine systems using 30.9 billion

111 metagenomic reads from the TARA Oceans Project (Sunagawa et al., 2015). Our

112 investigation revealed that closely related Prochlorococcus populations sharing the same

113 high-light niche (i.e., near the surface) exhibit considerable differences in their relative

114 abundance that could be explained by a small number of differentially occurring gene

115 clusters. Finally, we extended our analysis of 31 isolates with 74 single-amplified

116 genomes (SAGs) and revealed intriguing patterns within Prochlorococcus hypervariable

117 genomic islands by quantifying the link between individual gene clusters and the 118 environment

\section{Materials and methods}

120 The URL http://merenlab.org/data/2017_Delmont_and_Eren_Metapangenomics/

121 contains a reproducible workflow that extends the descriptions and parameters of

122 programs used in our study to (1) compute the Prochlorococcus pangenome using 31

123 isolate genomes, (2) profile reads isolate genomes recruited from metagenomes, and (3)

124 generate a metapangenome for Prochlorococcus.

125 Genomes and metagenomes. We acquired 31 isolate genomes and 74 SAGs

126 (minimum length $>1 \mathrm{Mbp}$ ) of Prochlorococcus from the National Center for Biotechnology

127 Information (NCBI). We downloaded 93 TARA Oceans metagenomes from the European

128 bioinformatics institute $(\mathrm{EBI})$ repositories using the project accession number 
129 ERP001736. Supplementary Table 1 reports accession numbers and other information

130 for each isolate genome, SAG and metagenome.

131 Data preparation, quality filtering, and read recruitment. We removed the low-quality

132 reads from the TARA Oceans dataset using 'iu-filter-quality-minoche', which is a program

133 in illumina-utils v1.4.1 (Eren et al., 2013) (available from

134 https://github.com/merenlab/illumina-utils), which implements the noise filtering

135 parameters described by Minoche et al. (Minoche, Dohm \& Himmelbauer, 2011). After

136 simplifying the header lines of 31 FASTA files for Prochlorococcus isolate genomes

137 using an anvi'o script 'reformat-fasta', we concatenated all FASTA files into a single file,

138 and used Bowtie2 (Langmead \& Salzberg, 2012) with default parameters and the

139 additional '--no-unal' flag to recruit quality-filtered short metagenomic reads on to

140 Prochlorococcus isolate genomes ('read recruitment' is an analogous term to 'mapping',

141 or 'short read alignment'). We used samtools (Li et al., 2009) to convert resulting SAM

142 files into sorted and indexed BAM files.

143 Phylogenomic analysis. We used Phylosift v1.0.1 (Darling et al., 2014) with default

144 parameters to quantify evolutionary distances between genomes. Briefly, Phylosift (1)

145 identifies a set of 37 marker gene families in each genome, (2) concatenates the

146 alignment of each marker gene family across genomes, and (3) computes a

147 phylogenomic tree from the concatenated alignment using FastTree 2.1 (Price, Dehal \&

148 Arkin, 2010). We finalized the phylogenomic tree by setting a midpoint root with FigTree

149 v.1.4.3 (Rambaut, 2009).

150 Analysis of metagenomic read recruitment. We used anvi'o (Eren et al., 2015) v3

151 (available from http://merenlab.org/software/anvio/) to profile the read recruitment results

152 following the workflow outlined by Eren et al. (Eren et al., 2015). Briefly, we first used the

153 program 'anvi-gen-contigs-database' to profile Prochlorococcus genomes, during which 
154 Prodigal v2.6.3 (Hyatt et al., 2010) with default settings identified open reading frames.

155 We used InterProScan v5.17-56 (Zdobnov \& Apweiler, 2001) and eggNOG-mapper

156 v0.12.6 (Huerta-Cepas et al., 2016) outputs for our genes with the program 'anvi-import-

157 functions' to import annotations from other databases, including PFAM (Bateman et al., 158 2004), and eggNOG (Jensen et al., 2008). We then used the program 'anvi-run-ncbi-

159 cogs' to annotate genes with functions by searching them against the December 2014 160 release of the Clusters of Orthologous Groups (COGs) database (Tatusov et al., 2000) 161 using blastp v2.3.0+ (Altschul et al., 1990). We finally used the program 'anvi-profile' to 162 process the BAM file and generate an anvi'o profile database, which stored the coverage 163 and detection statistics of each Prochlorococcus genome in the TARA Oceans data. We 164 used 'anvi-import-collection' to link contigs to genomes from which they originate. Finally, 165 the program 'anvi-summarize' generated a static HTML output that gave access to the 166 mean coverage values of each genome (and individual genes within them) across 167 metagenomes.

168 Operational definition of 'population'. In the context of our study we define 169 'population' as an agglomerate of naturally occurring microbial cells, genomes of which 170 are similar enough to align to the same genomic reference with high sequence identity 171 as defined by the read recruitment stringency. Therefore, we assume that the isolate 172 genomes in our study provide access to environmental populations to which they belong 173 through the recruitment of short metagenomic reads.

174 Criterion for 'detection'. Assessing the occurrence of low abundance genomes in

175 complex data accurately can be problematic due to non-specific recruitment of short 176 reads to regions that are conserved across multiple populations. For instance, although

177 Prochlorococcus populations are virtually absent from the Southern Ocean (Flombaum 178 et al., 2013), our genomes recruited up to $0.01 \%$ of the metagenomic reads from the 
179 Southern Ocean metagenomes matching to non-specific targets. To avoid high false-

180 detection rates, we assumed that a genome was 'detected' in a given metagenome only

181 if more than $50 \%$ of its nucleotide positions had coverage.

182 Classification of isolate genes as 'environmental core' and 'environmental

183 accessory'. Assuming the environmental niche of a population is defined by the

184 metagenomes in which it is 'detected', here we define 'environmental core genes' of a

185 population as the genes that are systematically detected in its niche. In contrast, the

186 genes that are not systematically detected within the niche of a given population

187 represent its environmental accessory genes. Genes in a population that are classified

188 as 'environmental core' given metagenomic data can be classified as 'accessory' given a

189 pangenome, and vice versa. To avoid any confusion between these operationally distinct

190 class designations, we refer to the genes classified given the metagenomic data as the

191 'environmental core genes' (ECGs), and the 'environmental accessory genes' (EAGs).

192 To identify ECGs and EAGs for each genome independently, we used the anvi'o script

193 'anvi-script-gen-distribution-of-genes-in-a-bin' with the parameter '--fraction-of-median-

194 coverage $0.25^{\prime}$. This script recovers the sum of coverage values for each gene in a given

195 genome across all metagenomes in which the population is 'detected', and marks the

196 genes that have less than $25 \%$ of the median coverage of all genes found in the genome

197 as EAGs. We then visualized resulting gene classes using the program 'anvi-interactive'.

198 Computing the pangenome, and the definition of gene clusters. The anvi'o

199 pangenomic workflow developed for this study consists of three major steps: (1)

200 generating an anvi'o genome database ('anvi-gen-genomes-storage') to store DNA and

201 amino acid sequences, as well as functional annotations of each gene in genomes

202 under consideration, (2) computing the pangenome ('anvi-pan-genome') from a genome

203 database by identifying 'gene clusters', and (3) displaying the pangenome ('anvi-display- 
204 pan') to visualize the distribution of gene clusters across genomes, interactively bin gene

205 clusters into logical groups, and inspect the alignment of genes in a given cluster 206 interactively. In our study, a 'gene cluster' represents sequences of one or more 207 predicted open reading frames grouped together based on their homology at the 208 translated DNA sequence level. Gene clusters with more than one sequence may 209 contain orthologous or paralogous sequences, or both, from one or more genomes

210 analyzed in the pangenome. To compute the Prochlorococcus pangenome, we first 211 generated an 'anvi'o genomes storage database' from the FASTA files of 31

212 Prochlorococcus isolate genomes using the '--internal-genomes' flag. We then used the

213 program 'anvi-pan-genome' with the genomes storage database, the flag '--use-ncbi-

214 blast', and parameters '--minbit 0.5', and '--mcl-inflation 10'. This program (1) calculates

215 similarities of each amino acid sequence in every genome against every other amino

216 acid sequence using blastp (Altschul et al., 1990), (2) removes weak hits using the

217 'minbit heuristic', which was originally described in ITEP (Benedict et al., 2014) to filter

218 weak hits based on the aligned fraction between the two reads, (3) uses the MCL

219 algorithm (Van Dongen \& Abreu-Goodger, 2012) to identify gene clusters in the

220 remaining blastp search results, (4) computes the occurrence of gene clusters across

221 genomes and the total number of genes they contain, (5) performs hierarchical

222 clustering analyses for gene clusters (based on their distribution across genomes) and

223 for genomes (based on gene clusters they share) using Euclidean distance and Ward

224 clustering by default, and finally (6) generates an anvi'o pan database that stores all

225 results for downstream analyses and can be visualized by the program 'anvi-display226 pan'.

227 Computing the metapangenome. Here we define 'metapangenome' as the outcome of 228 the analysis of pangenomes in conjunction with the environment where the abundance 229 and prevalence of gene clusters and genomes are recovered through shotgun 
230 metagenomes. To connect the environmental distribution patterns of genomes to the

231 Prochlorococcus pangenome, we used the program 'anvi-gen-samples-database' with

232 the genome coverage estimates reported in the summary of the anvi'o profile database

233 for metagenomic data. To quantify the ratio of 'environmental core genes' (ECGs) and

234 the 'environmental accessory genes' (EAGs) in each gene cluster in the resulting

235 pangenome, we used the anvi'o program 'anvi-script-gen-environmental-core-summary'

236 with default parameters. The program 'anvi-display-pan' visualized the Prochlorococcus

237 metapangenome, and 'anvi-summarize' generated a summary of gene clusters.

238 Analysis of Prochlorococcus single cell genomes. We performed a pangenomic

239 analysis combining the 74 SAGs and 31 isolate genomes of Prochlorococcus following

240 the same workflow as for the isolate genomes alone. From the 74 SAGs, we then

241 selected five phylogenetically distant ones and performed a metapangenomic analysis

242 following the same workflow as for the isolate genomes (including the same

243 metagenomic dataset). Our selection of few distant SAGs was intended to minimize the

244 dilution effect due to competing read recruitment onto identical regions from multiple

245 genomes.

246 Visualizations. We used the ggplot2 (Ginestet, 2011) library for $R$ to visualize the

247 relative distribution of genomic groups on the world map. Anvi'o performed all other

248 visualizations, and we finalized our figures for publication using Inkscape, an open-

249 source vector graphics editor (available from http://inkscape.org/).

250 Data availability. The TARA Oceans metagenomes are publicly available through the

251 European Bioinformatics Institute (accession IDs ERP001736). We also made available

252 (1) Prochlorococcus isolate genomes and SAGs (doi:10.6084/m9.figshare.5447221), (2)

253 the anvi'o database files and the static HTML summary output for Prochlorococcus

254 isolate genomes across TARA Oceans metagenomes 
255 (doi:10.6084/m9.figshare.5447224), (3) the metapangenome of Prochlorococcus

256 isolates (doi:10.6084/m9.figshare.5447227), an extended pangenome of

257 Prochlorococcus isolates and SAGs (doi:10.6084/m9.figshare.5447230), (4) and the

258 metapangenome of Prochlorococcus SAGs (doi:10.6084/m9.figshare.5447233). The

259 URL http://anvi-server.org/p/JNIBAB serves an interactive version of the

260 metapangenome of Prochlorococcus isolates.

\section{Results}

\section{Environmental distribution of Prochlorococcus isolate genomes}

263 To estimate the abundance and relative distribution patterns of the 31 Prochlorococcus

264 isolate genomes in environmental samples, we mapped to them 30.9 billion quality-

265 filtered metagenomic short reads from 93 TARA Oceans samples $(0.2-3 \mu \mathrm{m}$ planktonic

266 size fraction) that cover the Atlantic Ocean, Pacific Ocean, Indian Ocean, Southern

267 Ocean, Mediterranean Sea and Red Sea (Supplementary Table 1). Prochlorococcus

268 genomes recruited 1.68 billion reads $(5.44 \%$ of the dataset) from the surface $(0-15$

269 meters depth; $n=61$ ), and the subsurface chlorophyll maximum layer (17-95 meters

270 depth; $n=32$ ) metagenomes. The relative distribution of all Prochlorococcus genomes

271 ranged from below the detection limit in the Southern Ocean to $24.1 \%$ in a surface

272 metagenome from the Indian Ocean (Supplementary Table 2).

273 In agreement with the literature, genomes from the Clade LL-II and Clade LL-III were not

274 detected in the metagenomic dataset: although the isolation source for most LL-II/III

275 genomes were 120 meters (Rocap et al., 2002), the subsurface samples in TARA

276 Oceans metagenomes averaged 53.7 meters and never exceeded 100 meters. The

277 remaining clades displayed contrasting distribution patterns. The $\mathrm{HL}-\mathrm{I}$ and $\mathrm{HL}-\mathrm{II}$ genomes

278 were enriched in surface samples, but they were geographically antagonistic: HL-I 
279 dominated in the Mediterranean Sea, while HL-II, the most abundant Prochlorococcus

280 clade in the dataset, occurred mostly in the Indian Ocean and Red Sea (Figure S1).

281 Read recruitment results were also in line with previous observations suggesting

282 temperature as one of the main drivers of distribution patterns of $\mathrm{HL}-\mathrm{I}$ and $\mathrm{HL}-\mathrm{II}$

283 ((Johnson et al., 2006; Biller et al., 2014b) and references therein), as 93\% and 95\% of

284 the reads recruited by the HL-I and HL-II genomes originated from samples that were

285 below and above $22^{\circ} \mathrm{C}$, respectively. The LL-I and LL-IV genomes (more characteristic to

286 the subsurface layer) were also detected in different geographic locations, but in lower

287 proportions (Supplementary Table 2). Overall, the trends observed here are largely

288 consistent with results from previous environmental surveys and culture experiments

289 (Johnson et al., 2006; Larkin et al., 2016), and emphasize the limited niche overlap of

290 Prochlorococcus clades in the euphotic layer of marine systems on a large scale.

291 The pangenome of Prochlorococcus isolate genomes

292 Our pangenomic analysis of the 31 Prochlorococcus isolate genomes with a total of

29360,054 genes resulted in 7,385 gene clusters. We grouped these gene clusters into five

294 bins based on their occurrence across genomes: (1) HL+LL core gene clusters $(n=766)$,

295 (2) HL core gene clusters ( $n=492)$, (3) LL core gene clusters ( $n=144)$, (4) singletons (i.e.,

296 gene clusters associated with a single genome; $n=2,215)$, and (5) other gene clusters

297 that do not fit any of these classes $(n=3,768)$ (Figure S2). The singletons and HL+LL

298 core gene clusters corresponded to $30 \%$ and $10.4 \%$ of all clusters, respectively. This

299 relatively small core genome is consistent with previous pangenomic investigations and

300 supports the concept of a Prochlorococcus 'open pangenome' (Kettler et al., 2007).

$30149.1 \%$ of all clusters contained genes that were annotated with COG functions

302 (Supplementary Table 3). The functional annotation rate reached $90.5 \%$ for the HL+LL

303 core gene clusters. In contrast, it was only $37.2 \%$ for the singletons. As the shared gene 
304 content between genomes are effective predictors of their phylogenetic relationships

305 (Snel, Bork \& Huynen, 1999; Dutilh et al., 2004), we used the distribution of gene

306 clusters to determine the relationships among our genomes. The genomic groups that

307 emerged from this analysis matched the six Prochlorococcus phylogenetic clades

308 (Figure 1). However, a noticeable difference emerged from the organization of clades

309 based on gene clusters. Previous phylogenetic analyses using the internal transcribed

310 spacer region (Biller et al., 2014b) placed LL genomes into polyphyletic clades (LL-I

311 being an outlier), which was echoed by the phylogenomic analysis we performed in this

312 study using 37 core genes (Figure 1). In contrast, gene clusters grouped genomes

313 primarily based on their adaptation to light regimes (Figure 1). This result suggests that

314 employing the whole genomic content, instead of only marker genes, may be more

315 advantageous when the goal is to infer ecological rather than evolutionary relationships

316 between a set of closely related genomes.

317 Environmental core and accessory genes in Prochlorococcus isolate genomes

318 Genomic islands are widespread in Prochlorococcus (Coleman et al., 2006; Coleman \&

319 Chisholm, 2010) and genes from a given genome may not be found uniformly in all

320 marine ecosystems. Besides the detection estimates at the genome level, recruiting

321 reads from metagenomic data also provides an opportunity to investigate the occurrence

322 and relative distribution of individual genes. We used read recruitment statistics to

323 differentiate genes that co-occurred with the population across metagenomes from those

324 that consistently failed to recruit reads from the environment despite the occurrence of

325 the population. While the first group of genes is common to most cells in a given

326 population (i.e., connected to the environment), the second group of genes occurs only

327 in a fraction of the members of the population, or shows sporadic distribution patterns

328 across environments (i.e., not connected to the environment). This analysis revealed 
32942,777 environmental core genes (ECGs) and 6,528 environmental accessory genes

330 (EAGs) in 25 Prochlorococcus genomes (genomes from the Clade LL-II and Clade L-III

331 were not detected in the metagenomic data, hence did not yield any estimates)

332 (Supplementary Table 3$)$. The EAGs represented in average $13.4 \%( \pm 4.65 \%)$ of all

333 genes for each Prochlorococcus genome, exposing a non-negligible, and relatively

334 stable portion of genes occurring only in a small subset of the cells within each

335 population to which we had access through the genomic database and metagenomic

336 data, consistent with previous metagenomic surveys of this lineage (Coleman \&

337 Chisholm, 2010). The synteny of most EAGs in a given genome were not random, and

338 they mostly were clustered into hypervariable genomic islands (Figure 2). The

339 classification of the genes in an isolate genome based on their environmental

340 connectivity through metagenomics offers unique insights regarding their occurrence

341 within a population. Furthermore, this particular use of metagenomes is also essential to

342 subsequently quantify the environmental connectivity of genes in pangenomes.

343 The metapangenome reveals closely related isolates with different levels of

344 fitness

345 A metapangenome provides access to the environmental detection of individual genes in

346 gene clusters, along with the ecological niche boundaries of individual genomes. The

347 Prochlorococcus metapangenome revealed differences within the members of the Clade

$348 \mathrm{HL}-\mathrm{Il}$ with respect to their rate of detection in the environment (Figure 3; see the

349 interactive version at the URL http://anvi-server.org/p/JNIBAB). Interestingly, the

350 organization of genomes in $\mathrm{HL}-\mathrm{Il}$ based on gene clusters matched their detection

351 gradient within their niche, with the least abundant and the most abundant genomes in

352 the metagenomic data being at the two extremes of the cluster that described the Clade

$353 \mathrm{HL}-$ II (Figure 3, Supplementary Table 2). We tentatively grouped the HL-II genomes into 
354 three sub-groups based on their abundance in the metagenomic dataset: HL-II-Low

$355(n=3)$ with an average relative abundance of $0.037 \%$, HL-II-Medium $(n=10)$ with an

356 average relative abundance of $0.14 \%$, and $\mathrm{HL}-\mathrm{II}-\mathrm{High}(\mathrm{n}=4)$ with an average relative

357 abundance of $0.5 \%$. Based on this grouping, $\mathrm{HL}-\mathrm{II}-\mathrm{High}$ genomes were 13.5 times more

358 abundant in the environment on average compared to HL-II-Low genomes, despite being 359 closely related enough to be described in the same phylogenetic group for HL. In light of

360 this observation, we investigated whether the differentially distributed gene clusters

361 could identify the functional basis of the apparent change in fitness. Noticeably, the HL-II-

362 Low genomes were lacking gene clusters that resolve to DNA repair (DNA ligase; 3-

363 methyladenine DNA glycosylase; DEAD DEAH box helicase) compared to the HL-II-High

364 genomes (Supplementary Table 3). All 31 isolates carried DNA repair genes, as it is a

365 critical protection mechanism towards light induced damages occurring in the surface

366 layer of marine systems (Jeffrey et al., 1996); however, HL-II-High genomes carried a

367 unique set of DNA repair genes that were missing from HL-II-Low genomes. Also

368 missing from the HL-II-Low genomes were gene clusters corresponding to enzymes of

369 the cupin superfamily, the fructose-bisphosphate aldolase class II, glutamine amino

370 transferase, PAP fibrilin, a metal-binding protein, and 25 gene clusters to which we could

371 not assign a function. The metapangenome provided access to genomic features that

372 may explain the functional basis of such variation of fitness between closely related

373 members of the HL-II group. Assuming that an increased relative abundance in the

374 environment is equivalent to increased fitness, characterization of the genomic features

375 that contribute to these differences, especially those of unknown functions, warrants

376 further study.

377 Genes and functions connect the hypervariable genomic islands of

378 Prochlorococcus populations 
379 We then turned our attention to the key contribution of our metapangenomic workflow;

380 the environmental connectivity of the pangenome as defined by the proportion of ECGs

381 and EAGs found in each gene cluster. The percentage of EAGs from genomes that

382 occurred in our metagenomic data differed markedly between the $\mathrm{HL}+\mathrm{LL}$ core gene

383 clusters $(4.31 \%)$, LL core gene clusters $(0.28 \%)$, HL core gene clusters $(12.4 \%)$, and

384 singletons (66\%) (Figure 3; Supplementary Table 3). More than an order of magnitude

385 difference between the ratio of ECGs to EAGs among the LL and HL core gene clusters

386 suggests that, given the available isolate genomes, Prochlorococcus genes

387 characteristic to low-light regime may be more stable than those characteristic to high-

388 light regime. These results also indicate that genes present in all isolate genomes

389 ( $H L+L L$ core) were maintained in a large fraction of the cells in populations we

390 investigated, while those that are specific to a single isolate largely occurred in smaller

391 number of cells in the environment and remained below our detection limit. Exceptions to

392 low number of EAGs in $\mathrm{HL}+\mathrm{LL}$ core were gene clusters \#33, \#44 and \#431 (see

393 Supplementary Table 3). The percentage of EAGs for these gene clusters in HL isolates

394 were 100\%, 95.2\% and $95.2 \%$, and their functions resolved to 'nucleotide sugar

395 epimerase', 'udp-glucose 6-dehydrogenase' and 'mannose-1-phosphate

396 guanylyltransferase', respectively. In contrast, these gene clusters contained only ECGs

397 in the LL isolates (Supplementary Table 3). Sugar uptake by Prochlorococcus has been

398 observed in both culture and in situ (Gomez-Baena et al., 2008; Muñoz-Marín et al.,

$3992013,2017)$ and this process can support the growth of Prochlorococcus populations in

400 the surface ocean (Moisander et al., 2012). The occurrence of multiple sugar

401 metabolism genes in every $\mathrm{HL}$ isolate that are absent in almost all metagenome creates

402 an interesting conundrum.

403 To investigate whether this could be due to a cultivation bias that selects for members

404 from these populations with a certain set of sugar utilization genes, we analyzed 74 
405 single amplified genomes (SAGs) from a study by Kashtan et al. (Kashtan et al., 2014)

406 (Supplementary Table 4). Our analysis revealed that these gene clusters also occurred

407 in a large number of SAGs $(75.7 \%$ to $81.1 \%)$ (Supplementary Table 4). Most

408 interestingly, metapangenomic analysis of SAGs using the same metagenomic dataset

409 and bioinformatics workflow we used for the isolates also revealed that all genes in these

410 gene clusters were EAGs (Supplementary Table 4), consistent with our observations in

411 the $\mathrm{HL}$ isolates, and ruling out the 'cultivation bias' hypothesis. Yet, these results left us

412 with a puzzling observation as we have identified Prochlorococcus gene clusters

413 widespread in both isolate genomes and SAGs of the HL clades with genes rarely

414 detected in the surface oceans and seas. Methodological differences could explain the

415 conflict between the high prevalence of these gene clusters across genomes in the

416 pangenome and the low detection of each gene in them across metagenomes: gene

417 clusters are formed based on homology between amino acid sequences (Tettelin et al.,

418 2005), hence can contain genes with relatively low sequence similarity, while

419 metagenomic read recruitment is done at the DNA sequence-level, and is more 420 stringent.

421 Notably, genes in clusters \#33, \#44 and \#431 occurred in hypervariable genomic islands

422 of the isolates and SAGs (Figure 4, Supplementary Tables 3 and 4), and as a result are

423 surrounded by other EAGs that are not part of the Prochlorococcus core genome. To the

424 best of our knowledge this is the first time the Prochlorococcus core pangenome is

425 linked to hypervariable genomic islands, indicating that core functionalities of this major

426 lineage associated with sugar metabolism are maintained in a variety of versions within

427 each population. Finally, analyzing the functionality of all EAGs led us to expose a 428 prevalent role of sugar metabolism in hypervariable genomic islands beyond the three 429 core gene clusters (Figure 4 and S3). Briefly, functions such as udp-glucose 4430 epimerase, dTDP-4-dehydrorhamnose 3,5-epimerase, dTDP-4-dehydrorhamnose 
431 reductase, dTDP-glucose 4-6-dehydratase, GDP-mannose 4,6-dehydratase and

432 glucose-1-phosphate cytidylyltransferase were dominated by EAGs and occurred mostly

433 in hypervariable genomic islands of the HL populations (Supplementary Table 5).

434 Overall, our analyses exposed a high rate of gene diversification traits for sugar

435 metabolism in Prochlorococcus that might contribute to its remarkably high fitness in the

436 surface ocean.

\section{Discussion}

438 The quantity of data in genomic databases and metagenomic surveys is increasing

439 rapidly thanks to the advances in biotechnology and computation. Metapangenomes

440 take advantage of both genomes and metagenomes to link two important endeavors in

441 microbiology: inferring the relationships between isolate genomes through identifying the

442 core and accessory genes they harbor de novo, and investigating the relative distribution

443 of microbial populations and individual genes in the environment through metagenomics.

444 Our metapangenomic workflow has similarities to the method described in a recently

445 introduced metagenomics pipeline by Nahfach et al. (2016), as both efforts offer

446 solutions to expand conventional analyses of pangenomes by not only estimating the

447 abundance and distribution of gene clusters in the environment, but also linking them to

448 the distribution patterns of microbial populations. In addition to this shared goal, our

449 approach provides a flexible starting point with project-specific genomic databases

450 (rather than pre-computed references), and includes a comprehensive visualization

451 strategy to summarize metapangenomes.

452 The Prochlorococcus metapangenome revealed subtle distribution gradients among

453 isolates that belonged to the same phylogenetic clade, and exposed differentially

454 occurring gene clusters that could be related to genomic traits affecting the fitness 
455 among closely related members. It also revealed gene clusters that occurred in every

456 isolate genome and in most single-cell genomes but were largely missing in the

457 environment, exposing a core genome connecting hypervariable genomic islands of

458 distinct Prochlorococcus phylogenetic clades. Interestingly, these gene clusters were

459 biased towards sugar utilization. Variable genomic islands of Prochlorococcus among

460 co-occurring cells (Coleman et al., 2006) have previously been linked to the resistance of

461 viral infections (Avrani et al., 2011). Our findings here suggest that high sequence

462 diversification among genes involved in sugar metabolism may be beneficial for

463 Prochlorococcus populations, which should be further addressed. In addition, gene

464 clusters revealed that at least some of the genes in Prochlorococcus genomic islands

465 represent common functions with high rate of intra-population diversity at the DNA-level,

466 rather than recent horizontal transfers from other lineages. These observations

467 contribute to the ongoing debate on the origin, evolution and ecological role of

468 hypervariable genomic islands within microbial populations (Hacker \& Carniel, 2001;

469 Coleman et al., 2006; Wilhelm et al., 2007; Juhas et al., 2009; Fernández-Gómez et al.,

470 2012; Vineis et al., 2016). In addition to these novel insights, the parallels in our findings

471 and the extensive literature on Prochlorococcus emphasizes the potential of

472 metapangenomics to facilitate the recovery of key insights from novel and less studied

473 microbial populations, including those with no cultured representatives.

474 The vast majority of isolate and single cell genomes contain only a subset of the 475 complete set of genes microbial populations maintain within their niche boundaries

476 (Parkhill et al., 2000; Coleman et al., 2006; Juhas et al., 2009; Coleman \& Chisholm,

477 2010). Metagenomic data make it possible to classify genes in genomes based on their

478 occurrence in the environment. However, metagenomic short read recruitment alone

479 does not provide access to genes that are lacking in available genomes, even if they

480 may be critical for the functioning of the populations they originate. Characterizing all 
481 accessory genes of a given population in the environment is challenging due to the

482 limited coverage of the environmental metagenomes and genomic databases. These

483 limitations require careful interpretations of the observations that emerge from the

484 metapangenomic workflow and awareness that complete understanding of the

485 accessory genes of the environment may require additional efforts (Kashtan et al., 486 2014).

\section{Conclusion}

488 Here we developed novel software solutions and analytical tools within the open-source

489 software platform anvi'o to create and study metapangenomes with interactive

490 visualization and inspection capabilities. Our analysis of the Prochlorococcus

491 metapangenome revealed a small number of gene clusters that may be linked to subtle

492 fitness trends among very closely related members of this group, and displayed inter-

493 connectivity of hypervariable genomic islands across multiple clades. Our findings

494 suggest that metapangenomes can provide highly resolved linkage between core and

495 accessory genes of microbial populations and the environment, for any taxon and biome

496 for which genomic and metagenomic data are available, and can provide experimental

497 targets to explore the functional basis of niche partitioning and fitness. Besides isolate

498 and single-cell genomes, this strategy can also employ metagenome-assembled

499 genomes, and be used to study questions in the context of biotechnology or medicine.

\section{Acknowledgements}

501 We thank Bana Jabri, Sean Crosson, Ryan J. Newton, Maureen L. Coleman, Bas Dutilh,

502 Loïs Maignien, Julie Reveillaud, Michael D. Lee, and the members of the Meren Lab for

503 helpful discussions. We are also grateful to our anonymous reviewers for scrutinizing our

504 work, Özcan C. Esen for his technical insights and help, and Hilary G. Morrison for her 
505 guidance to improve our manuscript. Finally, we are in debt to the scientists who made

506 this study possible by generating the genomes and metagenomes, and making them

507 publicly available.

508 References

509

510

511

512

513

514

515

516

517

518

519

520

521

522

523

524

525

526

527

528

529

530

531

532

533

534

535

536

537

538

539

540

541

542

543

544

545

546

547

548

Al-Amoudi S., Razali R., Essack M., Amini MS., Bougouffa S., Archer JAC., Lafi FF., Bajic VB. 2016. Metagenomics as a preliminary screen for antimicrobial bioprospecting. Gene 594:248-258. DOI: 10.1016/j.gene.2016.09.021.

Alm RA., Ling LS., Moir DT., King BL., Brown ED., Doig PC., Smith DR., Noonan B., Guild BC., DeJonge BL., Carmel G., Tummino PJ., Caruso A., Uria-Nickelsen M., Mills DM., Ives C., Gibson R., Merberg D., Mills SD., Jiang Q., Taylor DE., Vovis GF., Trust TJ. 1999. Genomic-sequence comparison of two unrelated isolates of the human gastric pathogen Helicobacter pylori. Nature 397:176-180. DOI: 10.1038/16495.

Altschul SF., Gish W., Miller W., Myers EW., Lipman DJ. 1990. Basic local alignment search tool. Journal of molecular biology 215:403-410.

Anderson RE., Reveillaud J., Reddington E., Delmont TO., Eren AM., McDermott JM., Seewald JS., Huber JA. 2017. Genomic variation in microbial populations inhabiting the marine subseafloor at deep-sea hydrothermal vents. Nature Communications 8:1114. DOI: 10.1038/s41467-017-01228-6.

Avrani S., Wurtzel O., Sharon I., Sorek R., Lindell D. 2011. Genomic island variability facilitates Prochlorococcus-virus coexistence. Nature 474:604-8. DOI: 10.1038/nature10172.

Bateman A., Coin L., Durbin R., Finn RD., Hollich V., Griffiths-Jones S., Khanna A., Marshall M., Moxon S., Sonnhammer ELL., Studholme DJ., Yeats C., Eddy SR. 2004. @Pfam@The Pfam protein families database. Nucleic acids research 32:D138-41. DOI: $10.1093 / \mathrm{nar} / \mathrm{gkh} 121$.

Bendall ML., Stevens SLR., Chan LK., Malfatti S., Schwientek P., Tremblay J., Schackwitz W., Martin J., Pati A., Bushnell B., Froula J., Kang D., Tringe SG., Bertilsson S., Moran MA., Shade A., Newton RJ., McMahon KD., Malmstrom RR. 2016. Genome-wide selective sweeps and gene-specific sweeps in natural bacterial populations. ISME Journal 10:15891601. DOI: 10.1038/ismej.2015.241.

Benedict MN., Henriksen JR., Metcalf WW., Whitaker RJ., Price ND. 2014. ITEP: an integrated toolkit for exploration of microbial pan-genomes. BMC genomics 15:8. DOI: 10.1186/14712164-15-8.

Biller SJ., Berube PM., Berta-Thompson JW., Kelly L., Roggensack SE., Awad L., RoacheJohnson KH., Ding H., Giovannoni SJ., Rocap G., Moore LR., Chisholm SW. 2014a. Genomes of diverse isolates of the marine cyanobacterium Prochlorococcus. Scientific data 1:140034. DOI: $10.1038 /$ sdata.2014.34.

Biller SJ., Berube PM., Lindell D., Chisholm SW. 2014b. Prochlorococcus: the structure and function of collective diversity. Nature Reviews Microbiology 13:13-27. DOI: 10.1038/nrmicro3378.

Bork P., Bowler C., Vargas C de., Gorsky G., Karsenti E., Wincker P. 2015. Tara Oceans studies plankton at planetary scale. Science 348:873. DOI: 10.1126/science.aac5605.

Chisholm SW., Olson RJ., Zettler ER., Goericke R., Waterbury JB., Welschmeyer NA. 1988. A novel free-living prochlorophyte abundant in the oceanic euphotic zone. Nature 334:340343. DOI: $10.1038 / 334340 \mathrm{a} 0$. 
549

550

551

552

553

554

555

556

557

558

559

560

561

562

563

564

565

566

567

568

569

570

571

572

573

574

575

576

577

578

579

580

581

582

583

584

585

586

587

588

589

590

591

592

593

594

595

596

597
Coleman ML., Chisholm SW. 2010. Ecosystem-specific selection pressures revealed through comparative population genomics. Proc. Natl. Acad. Sci. Unit. States Am. 107:1863418639. DOI: 10.1073/pnas.1009480107/-/DCSupplemental.www.pnas.org/cgi/doi/10.1073/pnas.1009480 107.

Coleman ML., Sullivan MB., Martiny AC., Steglich C., Barry K., Delong EF., Chisholm SW. 2006. Genomic islands and the ecology and evolution of Prochlorococcus. Science (New York, N.Y.) 311:1768-1770. DOI: 10.1126/science.1122050.

Darling AE., Jospin G., Lowe E., Matsen FA., Bik HM., Eisen JA. 2014. PhyloSift: phylogenetic analysis of genomes and metagenomes. PeerJ 2:e243. DOI: 10.7717/peerj.243.

Delmont TO., Eren AM. 2016. Linking comparative genomics and environmental distribution patterns of microbial populations through metagenomics. bioRxiv:58750. DOI: $10.1101 / 058750$.

Delmont TO., Quince C., Shaiber A., Esen OC., Lee STM., Lucker S., Eren AM. 2017. NitrogenFixing Populations Of Planctomycetes And Proteobacteria Are Abundant In The Surface Ocean. bioRxiv.

Van Dongen S., Abreu-Goodger C. 2012. Using MCL to extract clusters from networks. Methods in Molecular Biology 804:281-295. DOI: 10.1007/978-1-61779-361-5_15.

Dutilh BE., Cassman N., McNair K., Sanchez SE., Silva GGZ., Boling L., Barr JJ., Speth DR., Seguritan V., Aziz RK., Felts B., Dinsdale EA., Mokili JL., Edwards RA. 2014. A highly abundant bacteriophage discovered in the unknown sequences of human faecal metagenomes. Nature communications 5:4498. DOI: 10.1038/ncomms5498.

Dutilh BE., Huynen MA., Bruno WJ., Snel B. 2004. The consistent phylogenetic signal in genome trees revealed by reducing the impact of noise. Journal of Molecular Evolution 58:527-539. DOI: 10.1007/s00239-003-2575-6.

Eren AM., Esen ÖC., Quince C., Vineis JH., Morrison HG., Sogin ML., Delmont TO. 2015. Anvi'o: an advanced analysis and visualization platform for 'omics data. PeerJ 3:e1319. DOI: $10.7717 /$ peerj. 1319 .

Eren AM., Vineis JH., Morrison HG., Sogin ML. 2013. A Filtering Method to Generate High Quality Short Reads Using Illumina Paired-End Technology. PLoS ONE 8:e66643. DOI: 10.1371/journal.pone.0066643.

Fernández-Gómez B., Fernàndez-Guerra A., Casamayor EO., González JM., Pedrós-Alió C., Acinas SG. 2012. Patterns and architecture of genomic islands in marine bacteria. BMC Genomics 13:347. DOI: 10.1186/1471-2164-13-347.

Fernández-Gómez B., Richter M., Schüler M., Pinhassi J., Acinas SG., González JM., PedrósAlió C. 2013. Ecology of marine Bacteroidetes: a comparative genomics approach. The ISME journal 7:1026-1037. DOI: 10.1038/ismej.2012.169.

Flombaum P., Gallegos JL., Gordillo RA., Rincon J., Zabala LL., Jiao N., Karl DM., Li WKW., Lomas MW., Veneziano D., Vera CS., Vrugt JA., Martiny AC. 2013. Present and future global distributions of the marine Cyanobacteria Prochlorococcus and Synechococcus. Proceedings of the National Academy of Sciences 110:9824-9829. DOI: 10.1073/pnas.1307701110.

Ginestet C. 2011. ggplot2: Elegant Graphics for Data Analysis. Journal of the Royal Statistical Society: Series A (Statistics in Society) 174:245-246. DOI: 10.1111/j.1467985X.2010.00676_9.x.

Gomez-Baena G., Lopez-Lozano A., Gil-Martinez J., Lucena JM., Diez J., Candau P., GarciaFernandez JM. 2008. Glucose Uptake and Its Effect on Gene Expression in Prochlorococcus. Plos One 3:e3416. DOI: 10.1371/journal.pone.0003416.

Hacker J., Carniel E. 2001. Ecological fitness, genomic islands and bacterial pathogenicity: A 
598

599

600

601

602

603

604

605

606

607

608

609

610

611

612

613

614

615

616

617

618

619

620

621

622

623

624

625

626

627

628

629

630

631

632

633

634

635

636

637

638

639

640

641

642

643

644

645

646
Darwinian view of the evolution of microbes. EMBO reports 2:376-381. DOI:

10.1093/embo-reports/kve097.

Handelsman J., Rondon MR., Brady SF., Clardy J., Goodman RM. 1998. Molecular biological access to the chemistry of unknown soil microbes: a new frontier for natural products. Chemistry \{\&\} Biology 5:R245--R249. DOI: 10.1016/S1074-5521(98)90108-9.

Haroon MF., Thompson LR., Parks DH., Hugenholtz P., Stingl U., Thompson LR., Churchill JH., Bower AS., McCorkle DC., Abualnaja Y., Sagar S., Jimenez-Infante F., Zhang G., Haroon MF., Zhang R., Hikmawan T., Stingl U., Fuller NJ., Qian P-Y., Ngugi DK., Stingl U., Rinke C., Brown CT., Grötzinger SW., Clingenpeel S., Clum A., Schwientek P., Rinke C., Woyke T., Gawad C., Koh W., Quake SR., Albertsen M., Nielsen HB., Sangwan N., Xia F., Gilbert JA., Haroon MF., Soo RM., Evans PN., Moore LR., Rocap G., Chisholm SW., Partensky F., Hess W., Vaulot D., Moore LR., Goericke R., Chisholm SW., Palenik B., Britschgi TB., Giovannoni SJ., Haroon MF., Thompson LR., Stingl U., Ivars-Martinez E., Santoro AE., DeLong EF., Sunagawa S., Bull AT., Stach JE., Markowitz VM., Rusch D., Schmieder R., Edwards R., Peng Y., Leung HC., Yiu S-M., Chin FY., Li H., Durbin R., Kang DD., Froula J., Egan R., Wang Z., Parks DH., Imelfort M., Skennerton CT., Hugenholtz P., Tyson GW., Eddy SR., Price MN., Dehal PS., Arkin AP., Felsenstein J., Dagan T., Roettger M., Bryant D., Martin W., Ludwig W., Letunic I., Bork P. 2016. A catalogue of 136 microbial draft genomes from Red Sea metagenomes. Scientific Data 3:160050. DOI: 10.1038/sdata.2016.50.

Huerta-Cepas J., Forslund K., Szklarczyk D., Jensen LJ., von Mering C., Bork P. 2016. Fast genome-wide functional annotation through orthology assignment by eggNOG-mapper. bioRxiv. DOI: 10.1101/076331.

Hyatt D., Chen G-L., Locascio PF., Land ML., Larimer FW., Hauser LJ. 2010. Prodigal: prokaryotic gene recognition and translation initiation site identification. BMC bioinformatics 11:119. DOI: 10.1186/1471-2105-11-119.

Jeffrey WH., Pledger RJ., Aas P., Hager S., Coffin RB., Von Haven R., Mitchell DL. 1996. Diel and depth profiles of DNA photodamage in bacterioplankton exposed to ambient solar ultraviolet radiation. Marine Ecology Progress Series 137:283-291. DOI: $10.3354 /$ meps 137283.

Jensen LJ., Julien P., Kuhn M., von Mering C., Muller J., Doerks T., Bork P. 2008. eggNOG: Automated construction and annotation of orthologous groups of genes. Nucleic Acids Research 36. DOI: 10.1093/nar/gkm796.

Johnson ZI., Zinser ER., Coe A., Mcnulty NP., Malcolm ES., Chisholm SW., Woodward EMS., Chisholm SW. 2006. Partitioning Among Prochlorococcus Ecotypes Along Environmental Gradients. Science 311:1737-1740. DOI: 10.1126/science.1118052.

Juhas M., Van Der Meer JR., Gaillard M., Harding RM., Hood DW., Crook DW. 2009. Genomic islands: Tools of bacterial horizontal gene transfer and evolution. FEMS Microbiology Reviews 33:376-393. DOI: 10.1111/j.1574-6976.2008.00136.x.

Kashtan N., Roggensack SE., Rodrigue S., Thompson JW., Biller SJ., Coe A., Ding H., Marttinen P., Malmstrom RR., Stocker R., Follows MJ., Stepanauskas R., Chisholm SW. 2014. SingleCell Genomics Reveals Hundreds of Coexisting Subpopulations in Wild Prochlorococcus. Science 344:416-420. DOI: 10.1126/science.1248575.

Kent AG., Dupont CL., Yooseph S., Martiny AC. 2016. Global biogeography of Prochlorococcus genome diversity in the surface ocean. The ISME Journal 10:1856-1865. DOI: 10.1038/ismej.2015.265.

Kettler GC., Martiny AC., Huang K., Zucker J., Coleman ML., Rodrigue S., Chen F., Lapidus A., Ferriera S., Johnson J., Steglich C., Church GM., Richardson P., Chisholm SW. 2007. Patterns and implications of gene gain and loss in the evolution of Prochlorococcus. PLoS 
647

648

649

650

651

652

653

654

655

656

657

658

659

660

661

662

663

664

665

666

667

668

669

670

671

672

673

674

675

676

677

678

679

680

681

682

683

684

685

686

687

688

689

690

691

692

693

694

695
Genetics 3:2515-2528. DOI: 10.1371/journal.pgen.0030231.

Kumar V., Sun P., Vamathevan J., Li Y., Ingraham K., Palmer L., Huang J., Brown JR. 2011. Comparative genomics of Klebsiella pneumoniae strains with different antibiotic resistance profiles. Antimicrobial agents and chemotherapy 55:4267-4276. DOI: 10.1128/AAC.0005211.

Langmead B., Salzberg SL. 2012. Fast gapped-read alignment with Bowtie 2. Nature methods 9:357-359. DOI: 10.1038/nmeth.1923.

Larkin AA., Blinebry SK., Howes C., Lin Y., Loftus SE., Schmaus CA., Zinser ER., Johnson ZI. 2016. Niche partitioning and biogeography of high light adapted Prochlorococcus across taxonomic ranks in the North Pacific. The ISME journal 10:1555-1567. DOI: 10.1038/ismej.2015.244.

Li H., Handsaker B., Wysoker A., Fennell T., Ruan J., Homer N., Marth G., Abecasis G., Durbin R. 2009. The Sequence Alignment/Map format and SAMtools. Bioinformatics (Oxford, England) 25:2078-2079. DOI: 10.1093/bioinformatics/btp352.

Lorenz P., Eck J. 2005. Metagenomics and industrial applications. Nature reviews. Microbiology 3:510-516. DOI: 10.1038/nrmicro1161.

Makarova K., Slesarev A., Wolf Y., Sorokin A., Mirkin B., Koonin E., Pavlov A., Pavlova N., Karamychev V., Polouchine N., Shakhova V., Grigoriev I., Lou Y., Rohksar D., Lucas S., Huang K., Goodstein DM., Hawkins T., Plengvidhya V., Welker D., Hughes J., Goh Y., Benson A., Baldwin K., Lee J-H., Díaz-Muñiz I., Dosti B., Smeianov V., Wechter W., Barabote R., Lorca G., Altermann E., Barrangou R., Ganesan B., Xie Y., Rawsthorne H., Tamir D., Parker C., Breidt F., Broadbent J., Hutkins R., O’Sullivan D., Steele J., Unlu G., Saier M., Klaenhammer T., Richardson P., Kozyavkin S., Weimer B., Mills D. 2006. Comparative genomics of the lactic acid bacteria. Proceedings of the National Academy of Sciences of the United States of America 103:15611-15616. DOI: 10.1073/pnas.0607117103.

Malmstrom RR., Coe A., Kettler GC., Martiny AC., Frias-Lopez J., Zinser ER., Chisholm SW. 2010. Temporal dynamics of Prochlorococcus ecotypes in the Atlantic and Pacific oceans. The ISME Journal 4:1252-1264. DOI: 10.1038/ismej.2010.60.

Minoche AE., Dohm JC., Himmelbauer H. 2011. Evaluation of genomic high-throughput sequencing data generated on Illumina HiSeq and genome analyzer systems. Genome biology 12:R112. DOI: 10.1186/gb-2011-12-11-r112.

Moisander PH., Zhang R., Boyle E a., Hewson I., Montoya JP., Zehr JP. 2012. Analogous nutrient limitations in unicellular diazotrophs and Prochlorococcus in the South Pacific Ocean. The ISME Journal 6:733-744. DOI: 10.1038/ismej.2011.152.

Muñoz-Marín M del C., Gómez-Baena G., Díez J., Beynon RJ., González-Ballester D., Zubkov M V., García-Fernández JM. 2017. Glucose uptake in Prochlorococcus: Diversity of kinetics and effects on the metabolism. Frontiers in Microbiology 8. DOI:

10.3389/fmicb.2017.00327.

Muñoz-Marín MDC., Luque I., Zubkov M V., Hill PG., Diez J., García-Fernández JM. 2013. Prochlorococcus can use the Pro1404 transporter to take up glucose at nanomolar concentrations in the Atlantic Ocean. Proceedings of the National Academy of Sciences of the United States of America 110:8597-602. DOI: 10.1073/pnas.1221775110.

Nayfach S., Rodriguez-Mueller B., Garud N., Pollard KS. 2016. An integrated metagenomics pipeline for strain profiling reveals novel patterns of bacterial transmission and biogeography. Genome research 26:1612-1625. DOI: 10.1101/gr.201863.115.

Olson RJ., Chisholm SW., Zettler ER., Altabet MA., Dusenberry JA. 1990. Spatial and temporal distributions of prochlorophyte picoplankton in the North Atlantic Ocean. Deep Sea Research Part A, Oceanographic Research Papers 37:1033-1051. DOI: 10.1016/0198- 
696

697

698

699

700

701

702

703

704

705

706

707

708

709

710

711

712

713

714

715

716

717

718

719

720

721

722

723

724

725

726

727

728

729

730

731

732

733

734

735

736

737

738

739

740

741

742

743

744
0149(90)90109-9.

Parkhill J., Wren BW., Mungall K., Ketley JM., Churcher C., Basham D., Chillingworth T., Davies RM., Feltwell T., Holroyd S., Jagels K., Karlyshev a V., Moule S., Pallen MJ., Penn CW., Quail M a., Rajandream M a., Rutherford KM., van Vliet a H., Whitehead S., Barrell BG. 2000. The genome sequence of the food-borne pathogen Campylobacter jejuni reveals hypervariable sequences. Nature 403:665-668. DOI: 10.1038/35001088.

Porter SS., Chang PL., Conow CA., Dunham JP., Friesen ML. 2016. Association mapping reveals novel serpentine adaptation gene clusters in a population of symbiotic Mesorhizobium. ISME Journal:1-15. DOI: 10.1038/ismej.2016.88.

Price MN., Dehal PS., Arkin AP. 2010. FastTree 2 - Approximately maximum-likelihood trees for large alignments. PLoS ONE 5. DOI: 10.1371/journal.pone.0009490.

Qin J., Li R., Raes J., Arumugam M., Burgdorf KS., Manichanh C., Nielsen T., Pons N., Levenez F., Yamada T., Mende DR., Li J., Xu J., Li S., Li D., Cao J., Wang B., Liang H., Zheng H., Xie Y., Tap J., Lepage P., Bertalan M., Batto JM., Hansen T., Le Paslier D., Linneberg A., Nielsen HB., Pelletier E., Renault P., Sicheritz-Ponten T., Turner K., Zhu H., Yu C., Jian M., Zhou Y., Li Y., Zhang X., Qin N., Yang H., Wang J., Brunak S., Doré J., Guarner F., Kristiansen K., Pedersen O., Parkhill J., Weissenbach J., Consortium M., Bork P., Ehrlich SD. 2010. A human gut microbial gene catalogue established by metagenomic sequencing. Nature 464:59-65.

Quince C., Delmont TO., Raguideau S., Alneberg J., Darling AE., Collins G., Eren AM. 2017. DESMAN: a new tool for de novo extraction of strains from metagenomes. Genome Biology 18:181. DOI: 10.1186/s13059-017-1309-9.

Rambaut A. 2009. FigTree, a graphical viewer of phylogenetic trees. Institute of Evolutionary Biology University of Edinburgh.

Read TD., Peterson SN., Tourasse N., Baillie LW., Paulsen IT., Nelson KE., Tettelin H., Fouts DE., Eisen JA., Gill SR., Holtzapple EK., Okstad OA., Helgason E., Rilstone J., Wu M., Kolonay JF., Beanan MJ., Dodson RJ., Brinkac LM., Gwinn M., DeBoy RT., Madpu R., Daugherty SC., Durkin AS., Haft DH., Nelson WC., Peterson JD., Pop M., Khouri HM., Radune D., Benton JL., Mahamoud Y., Jiang L., Hance IR., Weidman JF., Berry KJ., Plaut RD., Wolf AM., Watkins KL., Nierman WC., Hazen A., Cline R., Redmond C., Thwaite JE., White O., Salzberg SL., Thomason B., Friedlander AM., Koehler TM., Hanna PC., Kolstø A-B., Fraser CM. 2003. The genome sequence of Bacillus anthracis Ames and comparison to closely related bacteria. Nature 423:81-6. DOI: 10.1038/nature01586.

Reno ML., Held NL., Fields CJ., Burke P V., Whitaker RJ. 2009. Biogeography of the Sulfolobus islandicus pan-genome. Proceedings of the National Academy of Sciences of the United States of America 106:8605-10. DOI: 10.1073/pnas.0808945106.

Rocap G., Distel DL., Waterbury JB., Chisholm SW. 2002. Resolution of Prochlorococcus and Synechococcus ecotypes by using 16S-23S ribosomal DNA internal transcribed spacer sequences. Applied and Environmental Microbiology 68:1180-1191. DOI: 10.1128/AEM.68.3.1180-1191.2002.

Rocap G., Larimer FW., Lamerdin J., Malfatti S., Chain P., Ahlgren N a., Arellano A., Coleman M., Hauser L., Hess WR., Johnson ZI., Land M., Lindell D., Post AF., Regala W., Shah M., Shaw SL., Steglich C., Sullivan MB., Ting CS., Tolonen A., Webb E a., Zinser ER., Chisholm SW. 2003. Genome divergence in two Prochlorococcus ecotypes reflects oceanic niche differentiation. Nature 424:1042-1047. DOI: 10.1038/nature01947.

Rusch DB., Martiny AC., Dupont CL., Halpern AL., Venter JC. 2010. Characterization of Prochlorococcus clades from iron-depleted oceanic regions. Proceedings of the National Academy of Sciences of the United States of America 107:16184-16189. DOI: 10.1073 /pnas. 1009513107. 
745

746

747

748

749

750

751

752

753

754

755

756

757

758

759

760

761

762

763

764

765

766

767

768

769

770

771

772

773

774

775

776

777

778

779

780

781

782

783

784

785

786

787

788

789

790

791

792

793
Scholz M., Ward D V., Pasolli E., Tolio T., Zolfo M., Asnicar F., Truong DT., Tett A., Morrow AL., Segata N. 2016. Strain-level microbial epidemiology and population genomics from shotgun metagenomics. Nature Methods 13:435-438. DOI: 10.1038/nmeth.3802.

Sharon I., Morowitz MJ., Thomas BC., Costello EK., Relman DA., Banfield JF. 2013. Time series community genomics analysis reveals rapid shifts in bacterial species, strains, and phage during infant gut colonization. Genome research 23:111-120. DOI:

$10.1101 /$ gr.142315.112.

Smith DR., Doucette-Stamm LA., Deloughery C., Lee H., Dubois J., Aldredge T., Bashirzadeh R., Blakely D., Cook R., Gilbert K., Harrison D., Hoang L., Keagle P., Lumm W., Pothier B., Qiu D., Spadafora R., Vicaire R., Wang Y., Wierzbowski J., Gibson R., Jiwani N., Caruso A., Bush D., Reeve JN. 1997. Complete genome sequence of Methanobacterium thermoautotrophicum deltaH: functional analysis and comparative genomics. Journal of bacteriology 179:7135-55.

Snel B., Bork P., Huynen M a. 1999. Genome phylogeny based on gene content. Nature genetics 21:108-110. DOI: $10.1038 / 5052$.

Sunagawa S., Coelho LP., Chaffron S., Kultima JR., Labadie K., Salazar G., Djahanschiri B., Zeller G., Mende DR., Alberti A., Cornejo-Castillo FM., Costea PI., Cruaud C., D'Ovidio F., Engelen S., Ferrera I., Gasol JM., Guidi L., Hildebrand F., Kokoszka F., Lepoivre C., LimaMendez G., Poulain J., Poulos BT., Royo-Llonch M., Sarmento H., Vieira-Silva S., Dimier C., Picheral M., Searson S., Kandels-Lewis S., Bowler C., de Vargas C., Gorsky G., Grimsley N., Hingamp P., Iudicone D., Jaillon O., Not F., Ogata H., Pesant S., Speich S., Stemmann L., Sullivan MB., Weissenbach J., Wincker P., Karsenti E., Raes J., Acinas SG., Bork P. 2015. Ocean plankton. Structure and function of the global ocean microbiome. Science (New York, N.Y.) 348:1261359. DOI: 10.1126/science.1261359.

Tatusov RL., Galperin MY., Natale DA., Koonin E V. 2000. The COG database: a tool for genome-scale analysis of protein functions and evolution. Nucleic acids research 28:33-36. DOI: $10.1093 / \mathrm{nar} / 28.1 .33$.

Tettelin H., Masignani V., Cieslewicz MJ., Donati C., Medini D., Ward NL., Angiuoli S V., Crabtree J., Jones AL., Durkin AS., Deboy RT., Davidsen TM., Mora M., Scarselli M., Margarit y Ros I., Peterson JD., Hauser CR., Sundaram JP., Nelson WC., Madupu R., Brinkac LM., Dodson RJ., Rosovitz MJ., Sullivan SA., Daugherty SC., Haft DH., Selengut J., Gwinn ML., Zhou L., Zafar N., Khouri H., Radune D., Dimitrov G., Watkins K., O’Connor KJB., Smith S., Utterback TR., White O., Rubens CE., Grandi G., Madoff LC., Kasper DL., Telford JL., Wessels MR., Rappuoli R., Fraser CM. 2005. Genome analysis of multiple pathogenic isolates of Streptococcus agalactiae: implications for the microbial \&quot;pan-genome\&quot;. Proceedings of the National Academy of Sciences of the United States of America 102:13950-5. DOI: 10.1073/pnas.0506758102.

Thies S., Rausch SC., Kovacic F., Schmidt-Thaler A., Wilhelm S., Rosenau F., Daniel R., Streit W., Pietruszka J., Jaeger K-E. 2016. Metagenomic discovery of novel enzymes and biosurfactants in a slaughterhouse biofilm microbial community. Scientific Reports 6:27035. DOI: $10.1038 /$ srep27035.

Tringe SG., von Mering C., Kobayashi A., Salamov AA., Chen K., Chang HW., Podar M., Short JM., Mathur EJ., Detter JC., Bork P., Hugenholtz P., Rubin EM. 2005. Comparative metagenomics of microbial communities. Science (New York, NY) 308:554-557.

Tyson GW., Chapman J., Hugenholtz P., Allen EE., Ram RJ., Richardson PM., Solovyev V V., Rubin EM., Rokhsar DS., Banfield JF. 2004. Community structure and metabolism through reconstruction of microbial genomes from the environment. Nature 428:37-43. DOI: 10.1038 /nature 02340 .

Vineis JH., Ringus DL., Morrison HG., Delmont TO., Dalal S., Raffals LH., Antonopoulos DA., 
794

795

796

797

798

799

800

801

802

803

804

805

806

807

808

809

Rubin DT., Eren AM., Chang EB., Sogin ML. 2016. Patient-Specific Bacteroides Genome Variants in Pouchitis. mBio 7:e01713--16. DOI: 10.1128/MBIO.01713-16.

West NJ., Schönhuber WA., Fuller NJ., Amann RI., Rippka R., Post AF., Scanlan DJ. 2001. Closely related Prochlorococcus genotypes show remarkably different depth distributions in two oceanic regions as revealed by in situ hybridization using 16S rRNA-targeted oligonucleotides. Microbiology 147:1731-1744. DOI: 10.1099/00221287-147-7-1731.

Wilhelm LJ., Tripp HJ., Givan SA., Smith DP., Giovannoni SJ. 2007. Natural variation in SAR11 marine bacterioplankton genomes inferred from metagenomic data. Biology direct 2:27. DOI: $10.1186 / 1745-6150-2-27$.

Zdobnov EM., Apweiler R. 2001. InterProScan - an integration platform for the signaturerecognition methods in InterPro. Bioinformatics 17:847-848. DOI: 10.1093/bioinformatics/17.9.847.

Zhu C., Delmont TO., Vogel TM., Bromberg Y. 2015. Functional Basis of Microorganism Classification. PLoS computational biology 11:e1004472. DOI: 10.1371/journal.pcbi.1004472.

\section{Figure legends}

Figure 1: Organization of Prochlorococcus genomes based on the occurrence of gene clusters compared to phylogenomics. The dendrograms on the top shows the clustering of 31 isolate genomes based on the distribution of 7,385 gene clusters recovered from the pangenomic analysis (Euclidian distance and ward clustering). The tree at the bottom organizes the same genomes based on phylogenomics using 37 concatenated core genes. Colors indicate the phylogenetic affiliations of genomes based on published literature.

Figure 2: The gene-level detection of isolates from HL-I and HL-II in TARA Oceans metagenomes. Visualizations describe the gene-level niche partitioning of EQPAC1 and MIT9314, two isolates from the clade HL-I and HL-II, across 93 metagenomes from TARA Oceans. For each isolate, the genes are organized based on their order in the genome, and each layer corresponds to a metagenome, which are colored based on temperature $\left(<22^{\circ} \mathrm{C}\right.$ versus $>22^{\circ} \mathrm{C}$, accordingly to Figure 1$)$. The most outer layer describes the environmental connectivity of each gene. Environmental core and 
824 accessory genes are colored in green and red, respectively. Genomic sections enriched

825 in environmental accessory genes correspond to hypervariable regions.

826 Figure 3: The metapangenome of Prochlorococcus. Each one of the 7,385 gene

827 clusters contains one or more genes contributed by one or more isolate genomes. Bars

828 in the 31 first layers indicate the occurrence of gene clusters in a given isolate genome.

829 Gene clusters are organized based on their distribution across genomes (i.e., gene

830 clusters that co-occur in the same group of isolates are closer to each other), and

831 genomes are organized based on gene clusters they share using Euclidian distance and

832 ward ordination. The three next layers describe the gene clusters in which at least one

833 gene was functionally annotated using Pfams, EggNOGs, or COGs. Another layer

834 describes the ratio of environmental core versus environmental accessory genes

835 (ECGs/EAGs) within each PC. Gray areas account for the genes in genomes undetected

836 in the metagenomic dataset. Finally, the last layer corresponds to our selections of gene

837 clusters. The "HL+LL Core" selection corresponds to the gene clusters that contained

838 genes from all genomes. The "LL Core" and "HL Core" selections correspond to clusters

839 that contained genes characteristic to the LL- and HL-adapted genomes, respectively.

840 The last selection ("Singletons") corresponds to clusters that contained one or multiple

841 genes from a single genome. The right-hand side section of the figure provides

842 additional data for each isolate. The bottom rectangle displays the relative distribution of

843 genomes across 93 metagenomes and is followed by layers that show the average

844 distribution in the metagenomic dataset, number of genes from each isolate found in the

845 PC \#1, and the membership of isolates to phylogenetic clades. The dendrograms on the

846 top represents the hierarchical clustering of genomes based on the occurrence of gene 847 clusters. 
848 Figure 4: Prevalence of sugar utilization in Prochlorococcus hypervariable

849 genomic islands. Panel A describes the 25 most environmental accessory functions

850 identified in Prochlorococcus isolates defined by unusually high ratio of EAGs. Panels B

851 and $\mathrm{C}$ display the coordinates of genes corresponding to the 25 most environmental

852 accessory functions across five isolates genomes and five SAGs of Prochlorococcus,

853 respectively (red in the outer layers). Inner layers correspond to the 93 TARA Oceans

854 metagenomes, organized by geographic regions similarly to figure 2. For each

855 metagenome, black sections correspond to well covered genes while white sections

856 correspond to genes with no read recruitment.

\section{Supplementary figures and tables}

858 Figure S1: The distribution of isolates from HL-I and HL-II in TARA Oceans

metagenomes. World maps describe the cumulative relative distribution of

860 Prochlorococcus isolates from the clades HL-I (3 genomes) and HL-Il (17 genomes)

861 across 61 surface metagenomes. The size and color of dots varies as a function of

862 relative distributions and temperature range $\left(<22^{\circ} \mathrm{C}\right.$ versus $\left.>22^{\circ} \mathrm{C}\right)$, respectively.

863 Figure S2: The pangenome of Prochlorococcus. Each one of the 7,385 gene clusters

864 contains one or more genes contributed by one or more isolate genomes. Bars in the 31

865 horizontal layers indicate the occurrence of gene clusters in a given isolate genome.

866 Gene clusters are organized based on their distribution across genomes (i.e., gene

867 clusters that co-occur in the same group of isolates are closer to each other), and

868 genomes are organized based on gene clusters they share using Euclidian distance and

869 ward ordination. The "HL+LL Core" selection corresponds to the clusters that contained

870 genes from all genomes. The "LL Core" and "HL Core" selections correspond to gene

871 clusters that contained genes characteristic to the LL- and HL-adapted genomes, 
872 respectively. The last selection ("Singletons") corresponds to clusters that contained one

873 or multiple genes from a single genome.

874 Figure S3: Prevalence of sugar utilization in Prochlorococcus hypervariable

875 genomic islands. The figure displays the coordinates of genes corresponding to the 25

876 most environmental accessory functions across isolates genomes Prochlorococcus (red

877 in the outer layers). Inner layers correspond to the 93 TARA Oceans metagenomes,

878 organized by geographic regions similarly to figure 2 . For each metagenome, black

879 sections correspond to well covered genes while white sections correspond to genes

880 with no read recruitment. Genomes are organized based on gene clusters similarly to

881 Figure 3.

882 Supplementary Table 1: Summary of 31 Prochlorococcus genomes and 93

883 metagenomes from the TARA Oceans project.

884 Supplementary Table 2: Reads recruitments, detection and relative distribution of 31

885 Prochlorococcus genomes in 93 metagenomes from the TARA Oceans project.

886 Supplementary Table 3: Summary of the metapangenomic analysis of

887 Prochlorococcus isolates. The table describes the functionality and environment

888 connectivity of genes identified in the 31 Prochlorococcus isolate genomes, and links

889 each gene to a gene cluster in the Prochlorococcus pangenome.

890 Supplementary Table 4: Summary of the pangenomic and metapangenomic analyses

891 of Prochlorococcus isolates and SAGs. The table describes the pangenomic analysis of

89231 Prochlorococcus isolate genomes and 74 Prochlorococcus single cell genomes

893 (SAGs). The table also describes the metapangenome of five SAGs, which includes the

894 functionality and environment connectivity of genes and links each gene to a gene

895 cluster in the corresponding Prochlorococcus pangenome. 
896 Supplementary Table 5: Summary of the environmental connectivity of functions 897 identified in the 31 Prochlorococcus isolates. The table also links the environmental 898 connectivity of functions to the different clades of Prochlorococcus. 
Figure 1

Organization of Prochlorococcus genomes based on the occurrence of gene clusters compared to phylogenomics.

The dendrograms on the top shows the clustering of 31 isolate genomes based on the distribution of 7,385 gene clusters recovered from the pangenomic analysis (Euclidian distance and ward clustering). The tree at the bottom organizes the same genomes based on phylogenomics using 37 concatenated core genes. Colors indicate the phylogenetic affiliations of genomes based on published literature.

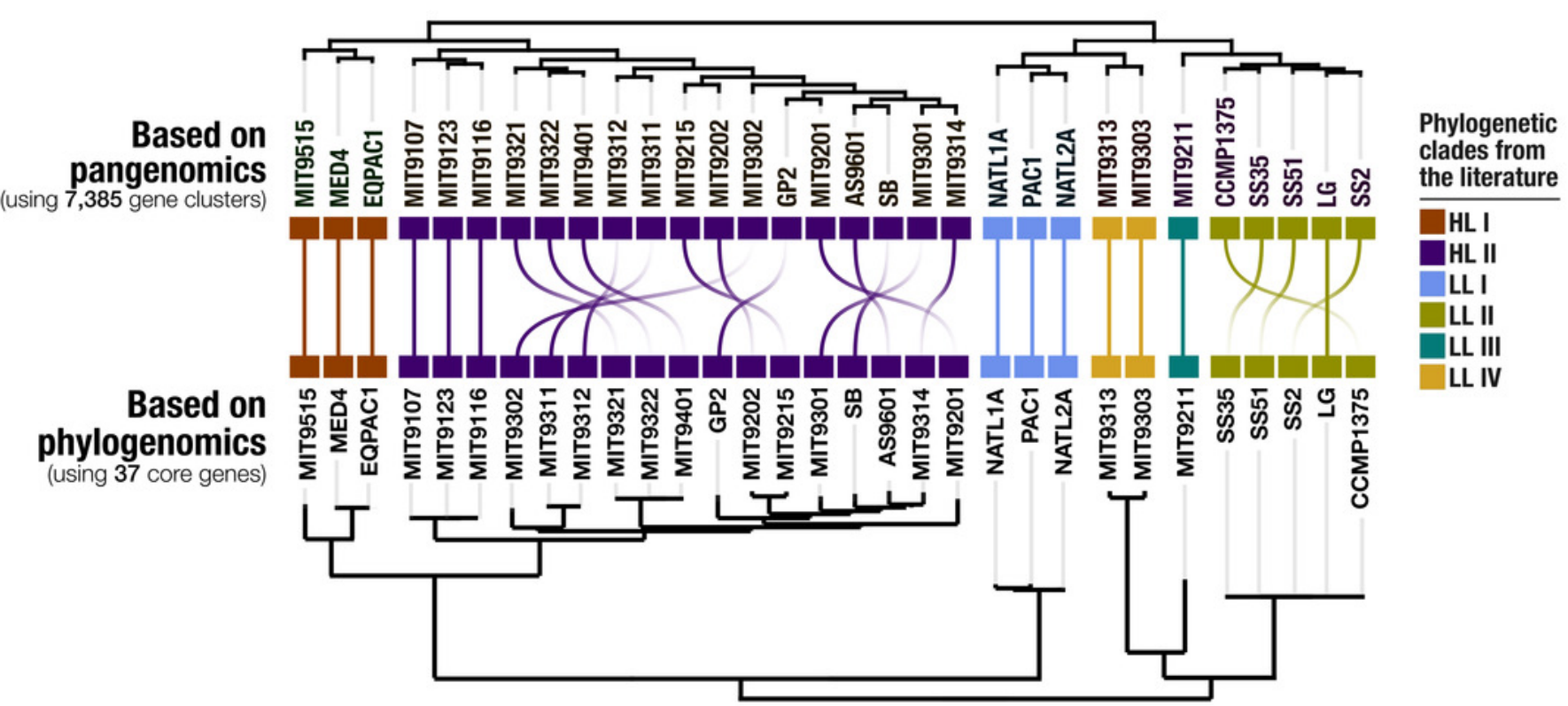




\section{Figure 2}

The gene-level detection of isolates from HL-I and HL-II in TARA Oceans metagenomes.

Visualizations describe the gene-level niche partitioning of EQPAC1 and MIT9314, two isolates from the clade HL-I and HL-II, across 93 metagenomes from TARA Oceans. For each isolate, the genes are organized based on their order in the genome, and each layer corresponds to a metagenome, which are colored based on temperature $\left(<22^{\circ} \mathrm{C}\right.$ versus $>22^{\circ} \mathrm{C}$, accordingly to Figure 1). The most outer layer describes the environmental connectivity of each gene.

Environmental core and accessory genes are colored in green and red, respectively. Genomic sections enriched in environmental accessory genes correspond to hypervariable regions.

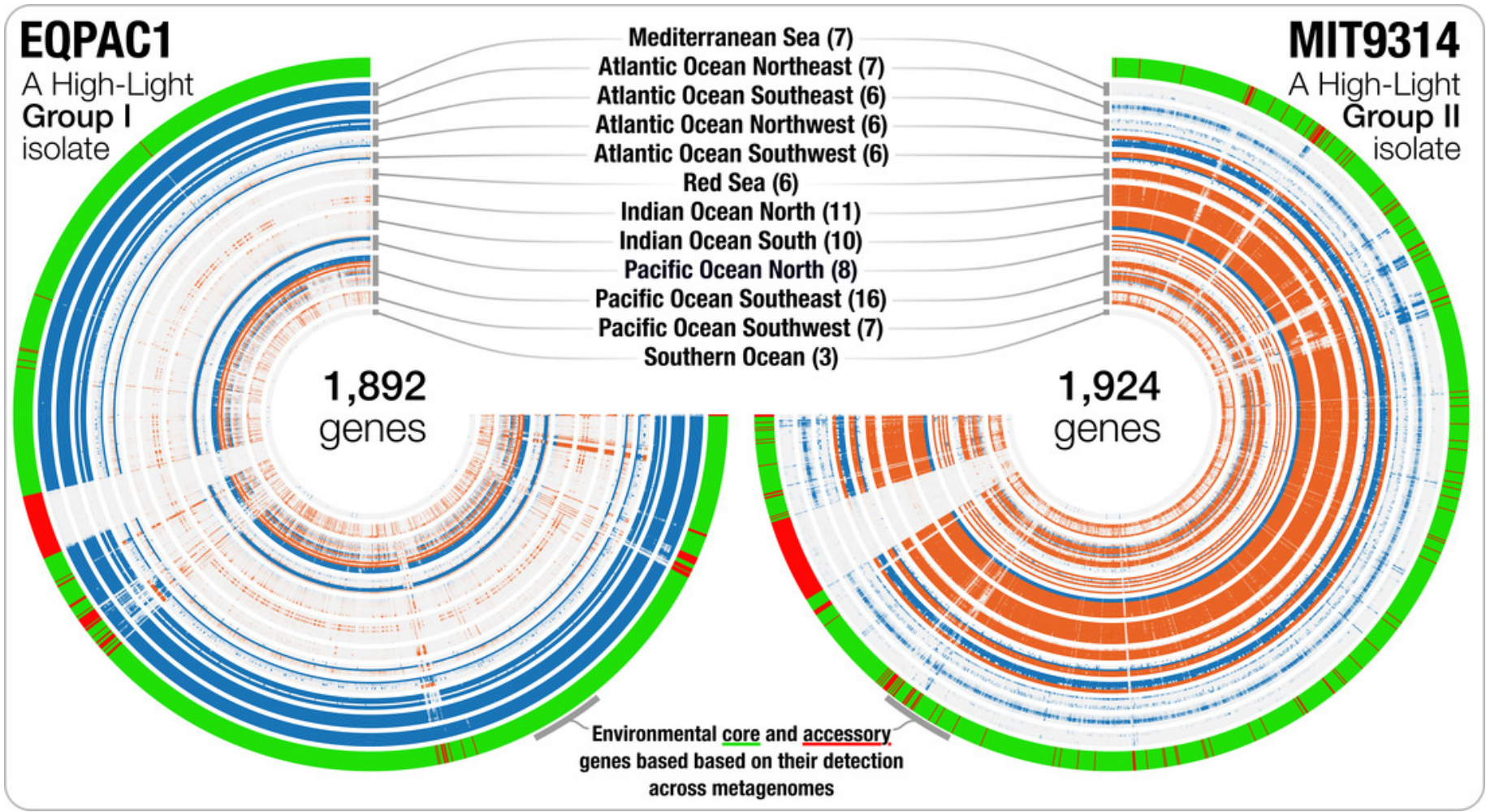




\section{Figure 3}

The metapangenome of Prochlorococcus.

Each one of the 7,385 gene clusters contains one or more genes contributed by one or more isolate genomes. Bars in the 31 first layers indicate the occurrence of gene clusters in a given isolate genome. Gene clusters are organized based on their distribution across genomes (i.e., gene clusters that co-occur in the same group of isolates are closer to each other), and genomes are organized based on gene clusters they share using Euclidian distance and ward ordination. The three next layers describe the gene clusters in which at least one gene was functionally annotated using Pfams, EggNOGs, or COGs. Another layer describes the ratio of environmental core versus environmental accessory genes (ECGs/EAGs) within each PC. Gray areas account for the genes in genomes undetected in the metagenomic dataset. Finally, the last layer corresponds to our selections of gene clusters. The "HL+LL Core" selection corresponds to the gene clusters that contained genes from all genomes. The "LL Core" and "HL Core" selections correspond to clusters that contained genes characteristic to the LL- and HL-adapted genomes, respectively. The last selection ("Singletons") corresponds to clusters that contained one or multiple genes from a single genome. The right-hand side section of the figure provides additional data for each isolate. The bottom rectangle displays the relative distribution of genomes across 93 metagenomes and is followed by layers that show the average distribution in the metagenomic dataset, number of genes from each isolate found in the PC \#1, and the membership of isolates to phylogenetic clades. The dendrograms on the top represents the hierarchical clustering of genomes based on the occurrence of gene clusters. 


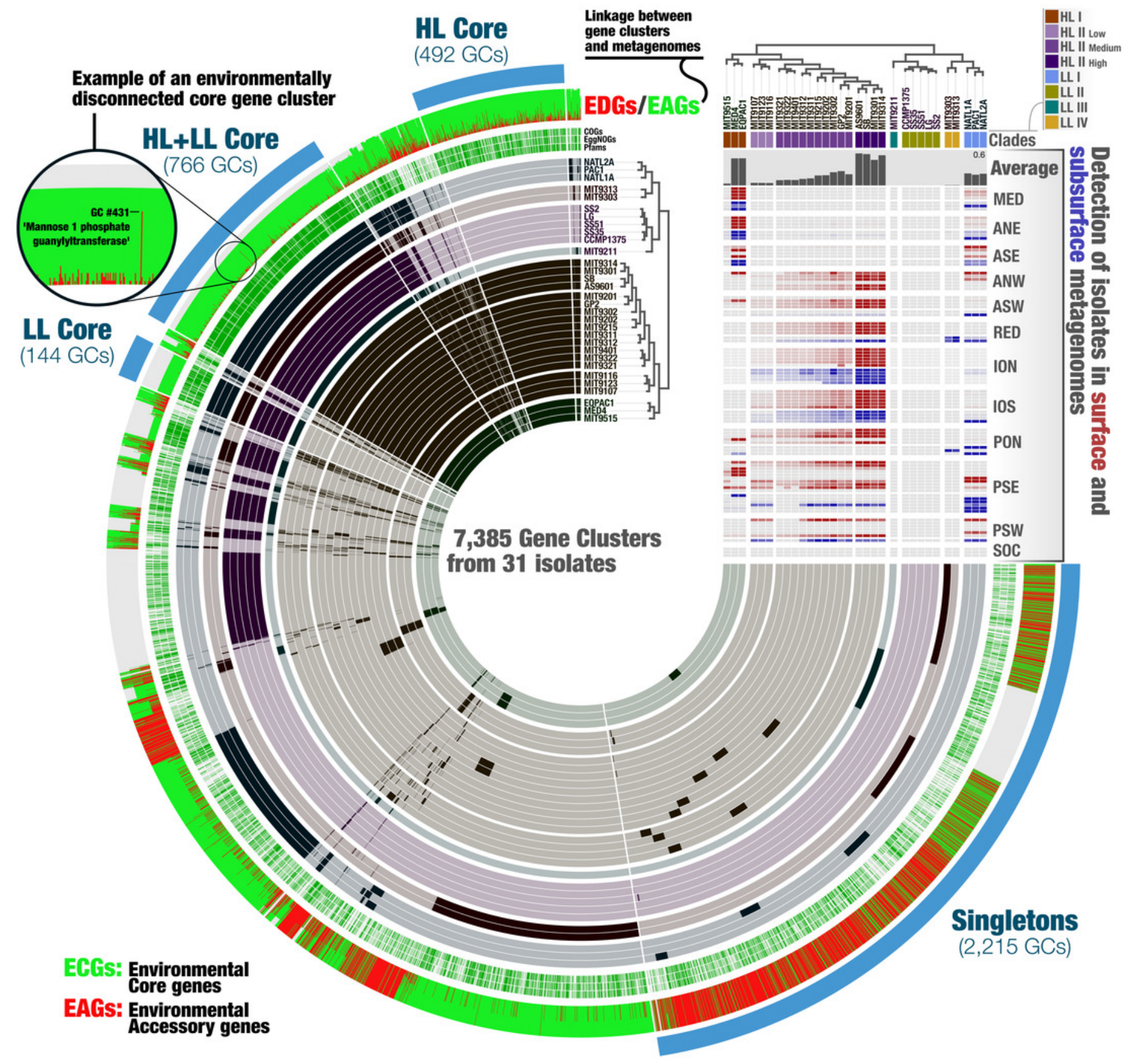




\section{Figure 4}

Prevalence of sugar utilization in Prochlorococcus hypervariable genomic islands.

Panel A describes the 25 most environmental accessory functions identified in Prochlorococcus isolates defined by unusually high ratio of EAGs. Panels B and C display the coordinates of genes corresponding to the 25 most environmental accessory functions across five isolates genomes and five SAGs of Prochlorococcus, respectively (red in the outer layers). Inner layers correspond to the 93 TARA Oceans metagenomes, organized by geographic regions similarly to figure 2 . For each metagenome, black sections correspond to well covered genes while white sections correspond to genes with no read recruitment. 

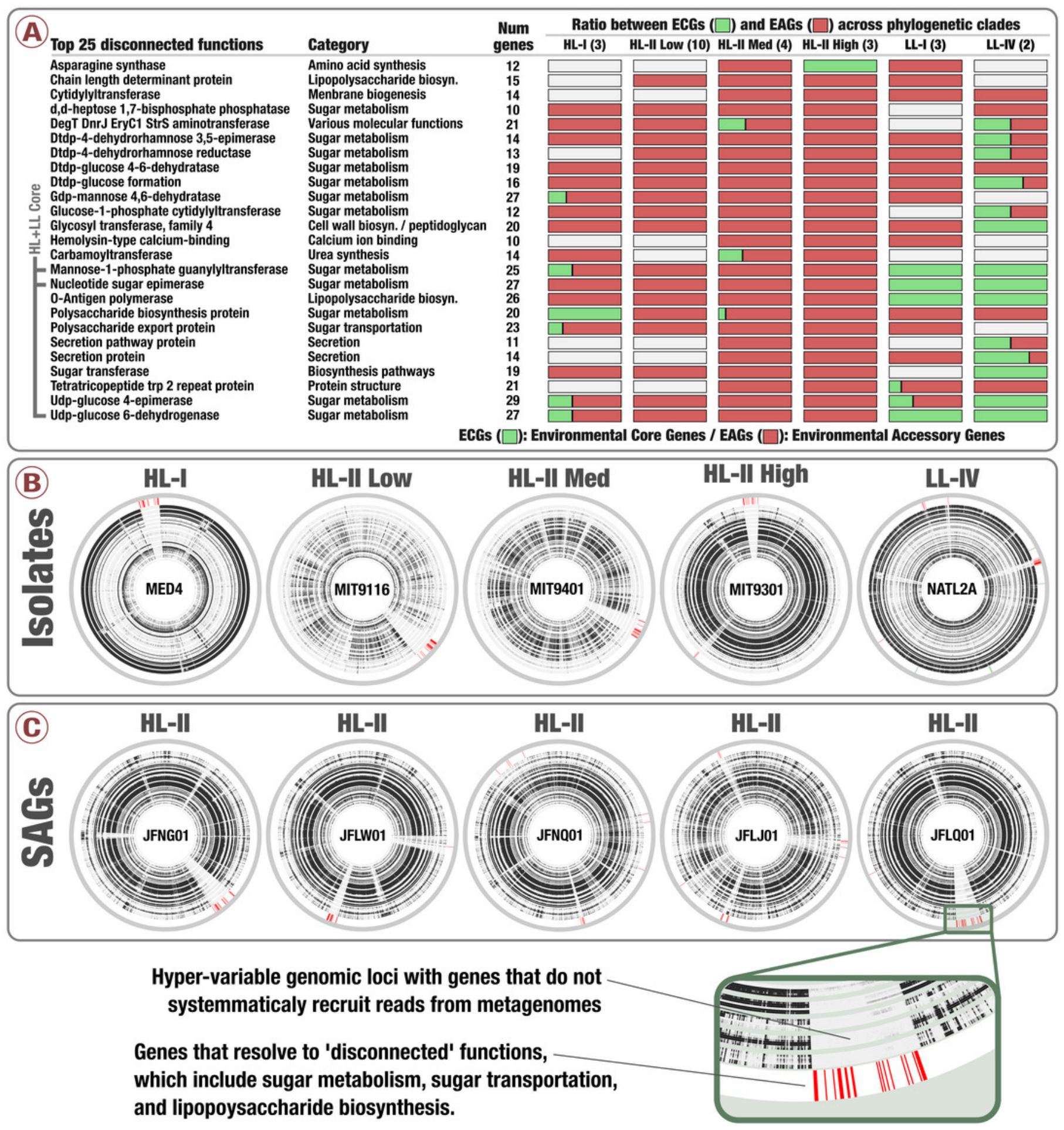